\title{
Impact of obstructive sleep apnea on new-set atrial fibrillation after septal myectomy in patients with hypertrophic obstructive cardiomyopathy
}

\author{
Jianbo Yu, Shengwei Wang, Changwei Ren, Hongchang Guo, Hehe Ma, Zhipeng Wei, Yongqiang Lai \\ Department of Cardiovascular Surgery Center, Beijing Anzhen Hospital, Capital Medical University, Beijing Institute of Heart, Lung and Blood \\ Vascular Diseases, Beijing, China \\ Contributions: (I) Conception and design: Y Lai; (II) Administrative support: Y Lai; (III) Provision of study materials or patients: Y Lai, J Yu; (IV) \\ Collection and assembly of data: S Wang, H Guo, H Ma, C Ren; (V) Data analysis and interpretation: J Yu, S Wang; (VI) Manuscript writing: All \\ authors; (VII) Final approval of manuscript: All authors. \\ Correspondence to: Yongqiang Lai, MD. No. 2, Anzhen Road, Chaoyang District, Beijing 100029, China. Email: yongqianglai@yahoo.com.
}

Background: Obstructive sleep apnea (OSA) is associated with a higher prevalence of postoperative atrial fibrillation in patients who underwent cardiac surgery. However, whether OSA is a risk factor for postoperative atrial fibrillation after septal myectomy remains unclear. We hypothesized that OSA was associated with postoperative atrial fibrillation after septal myectomy.

Methods: A total of 99 patients with hypertrophic obstructive cardiomyopathy who underwent septal myectomy were included in our manuscript. Polysomnography was performed in all patients, and the heart rhythm was continuously monitored during the perioperative period.

Results: In the present study, 25 (25.3\%) patients developed postoperative atrial fibrillation after septal myectomy. The prevalence of postoperative atrial fibrillation was significantly higher in patients with OSA and increased with the worsening severity of OSA. Notably, the apnea-hypoxia index was significantly higher in patients with postoperative atrial fibrillation among the different OSA groups. In receiver operating characteristic analysis, the area under the curve for the apnea-hypopnea index was 0.785 (95\% CI: 0.684$0.887, \mathrm{P}<0.001)$; an apnea-hypopnea index of 10.4 was the optimal cutoff point to predict postoperative atrial fibrillation. In the multivariable analysis, apnea-hypopnea index $\geq 10.4$ (odds ratio: 6.29 , 95\% CI: 2.18-18.14, $\mathrm{P}=0.001$ ), moderate-to-severe OSA (odds ratio: 4.88, 95\% CI: $1.42-16.86, \mathrm{P}=0.01$ ), and left atrium diameter (odds ratio: 1.12, 95\% CI: 1.03-1.22, $\mathrm{P}=0.01$ ) were independent risk factors associated with postoperative atrial fibrillation after adjusting for relevant variables. However, the association between the diagnosis of OSA and postoperative atrial fibrillation was no longer statistically significant.

Conclusions: The severity of OSA reflected by the apnea-hypopnea index in patients with obstructive hypertrophic cardiomyopathy who underwent surgery is an independent risk factor for postoperative atrial fibrillation, which is associated with adverse clinical outcomes.

Keywords: Obstructive sleep apnea (OSA); hypertrophic obstructive cardiomyopathy; postoperative atrial fibrillation (POAF); septal myectomy

Submitted Apr 11, 2021. Accepted for publication Jul 16, 2021.

doi: $10.21037 /$ jtd-21-632

View this article at: https://dx.doi.org/10.21037/jtd-21-632

(C) Journal of Thoracic Disease. All rights reserved. 


\section{Introduction}

Postoperative atrial fibrillation (POAF) is a common arrhythmic complication in patients who underwent cardiac surgery, including septal myectomy (1). According to different studies, the prevalence of POAF ranges from $17 \%$ to $25 \%(2,3)$. POAF has been regarded as a risk factor for adverse clinical outcomes in patients with obstructive hypertrophic cardiomyopathy (HOCM) who underwent septal myectomy, which is similar to findings in patients with preoperative AF (4). Therefore, it is important to evaluate patients to identify those who are at a high risk of $\mathrm{POAF}$ and require more attention during the perioperative period. Obstructive sleep apnea (OSA) is a common comorbidity in patients undergoing cardiac surgery, with a prevalence of mild and moderate-to-severe OSA of $74 \%$ and $48 \%$, respectively, which may predispose patients to postoperative complications $(5,6)$. Previous studies have reported that OSA is an independent predictor of POAF in patients who underwent cardiac surgery, including coronary artery bypass grafting, aortic valve replacement, mitral valve replacement/repair, or combined valve/coronary artery bypass grafting (7). In addition, the prevalence of OSA is high in patients with HOCM and positively associated with $\mathrm{AF}$ in HOCM patients $(8,9)$. However, it is unclear whether OSA is associated with POAF in patients who underwent septal myectomy. Therefore, we prospectively studied the relationship between OSA and POAF in these patients and hypothesized that OSA is associated with POAF after septal myectomy.

We present the following article in accordance with the STROBE reporting checklist (available at https://dx.doi. org/10.21037/jtd-21-632).

\section{Methods}

\section{Study population}

We prospectively studied 99 consecutive patients diagnosed with HOCM who underwent septal myectomy between July 2019 and November 2020 at Anzhen Hospital in Beijing. The diagnosis of HOCM and indications for septal myectomy were consistent with the 2011 AHA and 2014 ESC guidelines, which mainly included unexplained septal hypertrophy with a thickness of more than $15 \mathrm{~mm}(10,11)$. In addition, we excluded patients who met the following criteria: (I) had additional risk for on-pump cardiac surgery; (II) aged <18 years; (III) previous septal reduction therapy (septal myectomy or alcohol septal ablation); or (IV) previous history of paroxysmal or permanent $\mathrm{AF}, \mathrm{AF}$ surgical or catheter ablation, atrial flutter, or other types of atrial tachycardia. All patients underwent polysomnography (PSG) before the septal myectomy.

All patients provided informed consent, including biomarker and clinical data analysis, prior to enrollment. The study was approved by the Ethics Committee of Anzhen Hospital (Ethic committee study number: 2020034X). The study was conducted in accordance with the ethical principles of the Declaration of Helsinki (as revised in 2013).

\section{Echocardiography}

All patients underwent transthoracic echocardiography by an experienced physician using an E9 ultrasound machine. The diameters of the cardiac chambers were expressed as the maximum anteroposterior diameter in cardiac cycles. The thicknesses of the interventricular septum and ventricular wall were determined during diastole. In addition to the maximum thickness, the representative interventricular septal thickness was also recorded to determine overall thickness. The left ventricular outflow tract (LVOT) gradient was calculated using the simplified Bernoulli equation. In addition, the measurement of the LVOT gradient included both resting and induced conditions. Left ventricular ejection fraction and left atrial diameter were measured following the American Society of Echocardiography recommendations (12). Furthermore, mitral regurgitation was classified as mild, moderate, or severe.

\section{Polysomnography}

In the present study, all patients underwent standard PSG (Embletta; Embla, UK). OSA was defined as the absence of oro-nasal airflow for at least $10 \mathrm{~s}$ in the presence of outof-phase thoracoabdominal effort. The data were analyzed by an experienced scorer. The total recording time was used as the denominator to calculate the apnea-hypopnea index (AHI). AHI was calculated as the mean number of apneas and hypopneas per hour of sleep. If $5 \leq \mathrm{AHI} \leq 15$, the diagnosis was mild OSA. If $15<\mathrm{AHI} \leq 30$, the diagnosis was moderate OSA (13). In addition, if the AHI was $>30$, the diagnosis was severe OSA. Furthermore, we combined moderate and severe OSA to form the category moderateto-severe OSA in the present study. 


\section{Cardiac surgery and diagnosis of POAF}

All patients underwent septal myectomy in the present study. First, a standard median sternotomy was performed, and cardiopulmonary bypass was performed using ascending aortic cannulation and bicaval cannulation. Myocardial protection was achieved with an antegrade histidinetryptophan-ketoglutarate solution. The resection ranges were based on our previous study. Mitral intervention was not routinely conducted unless severe regurgitation or systolic anterior motion remained after myectomy. Concomitant procedures were appropriately performed according to the preoperative evaluation and intraoperative exploration. Detailed information is provided in our previous study (14).

All patients underwent continuous cardiac monitoring using a 5-lead telemetry strip during the perioperative period. The standard 12-lead electrocardiogram was performed daily. POAF was defined as an episode of $\mathrm{AF}$ lasting for more than $5 \mathrm{~min}$ or requiring antiarrhythmic therapy or electrical cardioversion after septal myectomy. Additional 12-lead electrocardiograms and Holter monitoring were performed to confirm the diagnosis of POAF when necessary.

\section{Statistical analysis}

The results are shown as mean \pm standard deviation, median (interquartile range), or percentage, when appropriate. The $\chi^{2}$ test was used to compare nominal variables among groups, as appropriate. Differences between the three groups were compared using one-way analysis of variance or the Kruskal-Wallis $\mathrm{H}$ test. Univariate and stepwise multivariate logistic regression analyses were used to identify the factors correlated with POAF. Variables with a $\mathrm{P}$ value $<0.10$ in univariate analysis were entered into multivariate logistic regression analysis. All reported probability values were two-tailed, and a $\mathrm{P}$ value $<0.05$ was considered statistically significant. SPSS version 26.0 software (IBM Corp., Armonk, NY, USA) and R 3.5.0 were used for calculations and illustrations, respectively.

\section{Results}

\section{Baseline patient characteristics of the study population}

We enrolled 99 patients diagnosed with HOCM who underwent septal myectomy (60 men and 39 women), and OSA was present in $56(56.6 \%)$ patients in our study (34 with mild OSA and 22 with moderate-to-severe OSA). The mean age of the study population was $48.8 \pm 13.0$ years. As shown in Table 1, patients with OSA were older than patients without OSA $(42.0 \pm 12.3$ vs. $54.9 \pm 11.5$ vs. $52.5 \pm 10.6$ years, $\mathrm{P}<0.001)$. Fasting glucose level and prevalence of syncope were significantly higher in patients with OSA. Furthermore, echocardiographic variables, including left atrial diameter, left ventricular end diastolic diameter, and number of moderate or severe mitral regurgitations, were significantly higher in patients with OSA. The PSG findings are shown in Table 1. Compared with those in patients without OSA, the AHI, oxygen desaturation index, and proportion of time with $\mathrm{SpO}_{2}<90 \%$ were higher in patients with OSA, while the mean $\mathrm{pO}_{2}$ level was lower in patients complicated with OSA. However, no difference was noted in the total sleep time between groups. Detailed information regarding the baseline characteristics is shown in Table 1.

\section{Perioperative data}

The perioperative data for the entire population are summarized in Table 2. There was no difference in most data, including the duration of cardiopulmonary bypass, aortic cross-clamp, postoperative ventilation, and length of ICU stay. However, patients with OSA had a higher prevalence of mitral valve intervention in the present study [10 (23.3\%) vs. 10 (29.4\%) vs. 12 (54.5\%), $\mathrm{P}=0.02]$. Furthermore, the 30-day mortality and rate of pacemaker implantation did not differ between the three groups. In the present study, 25 (25.3\%) patients developed POAF. Notably, the prevalence of POAF was significantly higher in patients with OSA and increased with the severity of OSA (Figure 1).

\section{Comparison of clinical variables between patients with and without POAF}

The clinical variables between patients with and without POAF are shown in Table 3. Compared to patients without POAF, age $(47.0 \pm 13.3$ vs. $54.0 \pm 10.9$ years, $\mathrm{P}=0.02)$ and left atrium diameter $(43.6 \pm 6.4$ vs. $47.6 \pm 5.9 \mathrm{~mm}, \mathrm{P}=0.007)$ were significantly higher in patients with POAF. However, there was no difference in the sex ratio between the two groups. Furthermore, the AHI, oxygen desaturation index, and mean $\mathrm{pO}_{2}$ during sleep were significantly higher in patients with OSA than in those without POAF. Most importantly, the AHI was significantly higher in patients with POAF among the different OSA groups (Figure 2). In addition, 
Table 1 Baseline characteristics of the study population

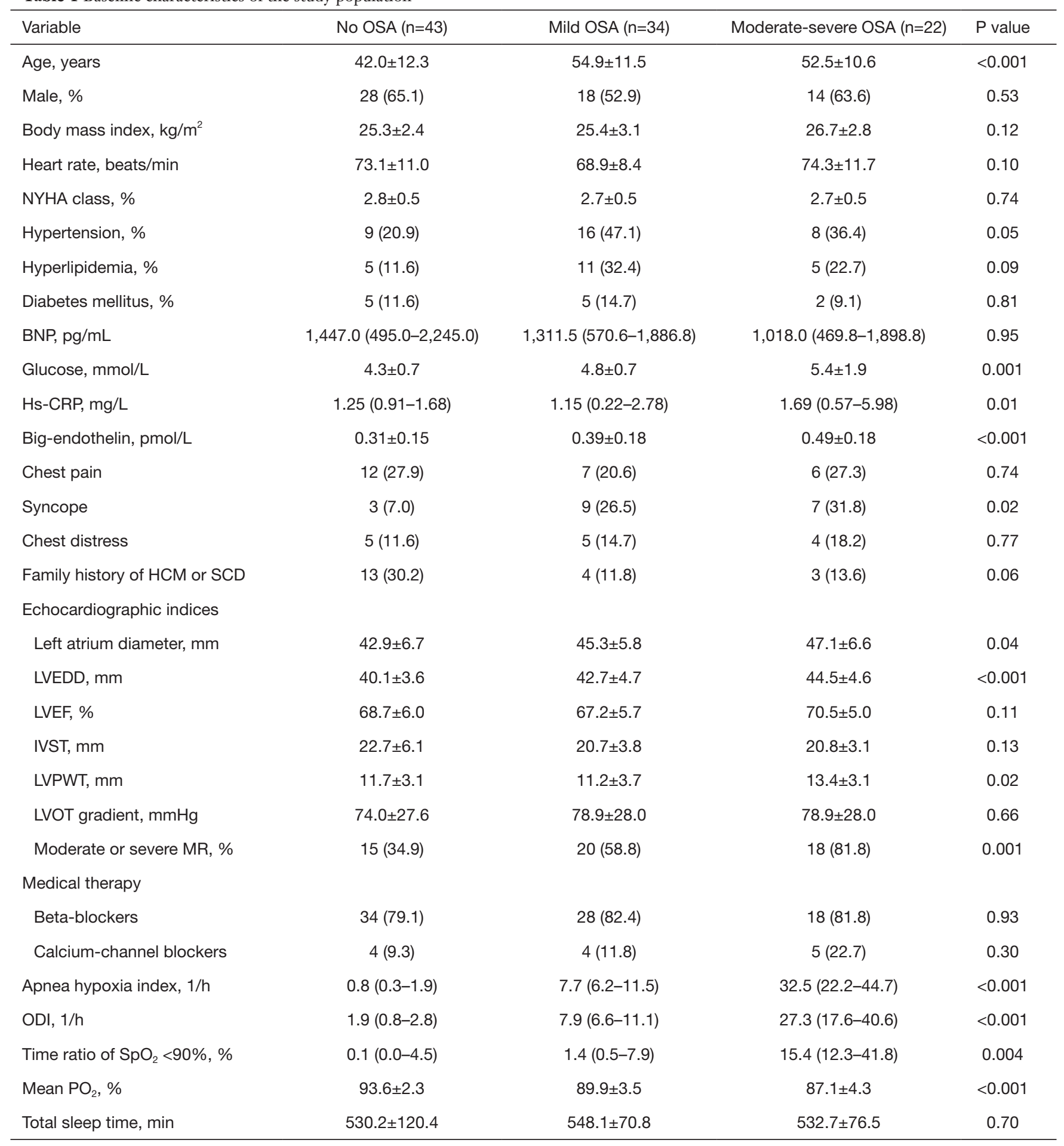

Values are presented as percentage, mean $\pm \mathrm{SD}$, or median (interquartile range) when appropriate. OSA, obstructive sleep apnea; HCM, hypertrophic myocardiopathy; SCD, sudden cardiac death; NYHA, New York Heart Association; hs-CRP, high-sensitivity C-reactive protein; BNP, brain natriuretic peptide; IVST, interventricular septal thickness; LVEF, left ventricular ejection fraction; LVEDD, left ventricular end diastole diameter; LVPWT, left ventricular posterior wall thickness; LVOT, left ventricular outflow tract; MR, mitral regurgitation; ODI, oxygen desaturation index. 
Table 2 Perioperative data

\begin{tabular}{|c|c|c|c|c|}
\hline Variable & No OSA $(n=43)$ & Mild OSA $(n=34)$ & Moderate-severe OSA $(n=22)$ & $P$ value \\
\hline Aortic cross-clamp time, min & $72.8 \pm 41.4$ & $65.9 \pm 25.7$ & $84.2 \pm 44.1$ & 0.25 \\
\hline Postoperative ventilation, $\mathrm{h}$ & $20.7 \pm 18.4$ & $22.0 \pm 7.6$ & $20.6 \pm 13.2$ & 0.91 \\
\hline Length of ICU stay, $\mathrm{h}$ & $54.6 \pm 25.5$ & $58.4 \pm 33.6$ & $49.4 \pm 31.8$ & 0.66 \\
\hline Mitral valve replacement or repair, \% & $10(23.3)$ & $10(29.4)$ & $12(54.5)$ & 0.02 \\
\hline 30-day mortality, \% & $0(0)$ & $1(2.9)$ & $0(0)$ & 0.38 \\
\hline Pacemaker implantation, \% & $1(2.3)$ & $2(5.9)$ & $0(0)$ & 0.43 \\
\hline POAF, \% & $6(14.0)$ & $8(23.5)$ & $11(50.0)$ & 0.002 \\
\hline
\end{tabular}

Values are presented as percentage, mean \pm SD, or median (interquartile range) when appropriate. OSA, obstructive sleep apnea; CPB, cardiopulmonary bypass; ICU, intensive care unit; CABG, coronary artery bypass graft; POAF, postoperative atrial fibrillation.

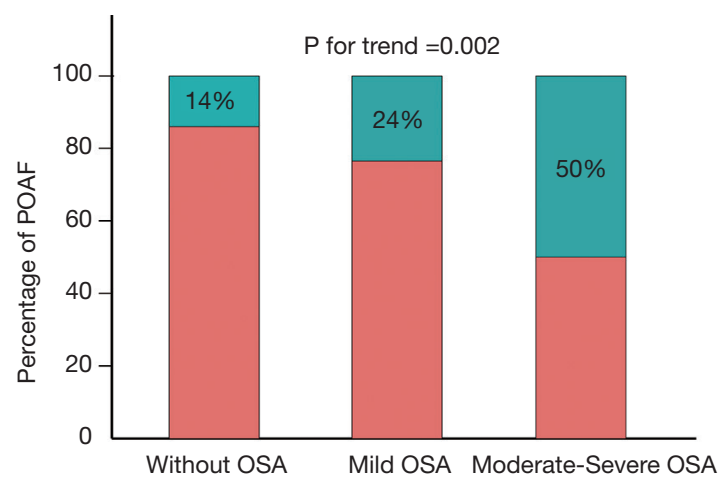

Figure 1 Prevalence of POAF as the severity of OSA. Prevalence of POAF increased with the severity of OSA. POAF, postoperative atrial fibrillation; OSA, obstructive sleep apnea.

patients with POAF had a higher prevalence of moderate or severe mitral regurgitation and concomitant coronary artery bypass graft than patients without POAF.

\section{Preoperative predictors of new-onset POAF}

We performed receiver operating characteristic curve analysis to assess the ability of AHI to identify patients who developed POAF. The area under the curve for AHI was 0.785 (95\% CI: 0.684-0.887; $\mathrm{P}<0.001$ ). The optimal cutoff value for AHI (10.4) to predict POAF had a sensitivity of 0.72 and specificity of 0.80 . Univariate and multivariate logistic regression analyses were used to evaluate the risk factors for POAF. In the univariate analysis, variables including age, left atrium diameter, interventricular septal thickness, moderate or severe mitral regurgitation, oxygen desaturation index, and mean $\mathrm{pO}_{2}$ during sleep were risk factors for POAF. Importantly, AHI $\geq 10.4$, severity of OSA, and presence of OSA were also risk factors for POAF. Furthermore, we conducted a multivariable logistic regression analysis to identify independent risk factors associated with POAF. In the multivariable analysis, we found that $\mathrm{AHI} \geq 10.4$ (Model 1), severity of OSA (Model 2), and left atrium diameter were independent risk factors associated with POAF after adjusting for age, sex, body mass index (BMI), and other relevant variables identified in the univariate analyses. However, after adjusting for age, sex, $\mathrm{BMI}$, and other relevant variables (Model 3), the association between the diagnosis of OSA and POAF was no longer significant. Detailed information was shown in Table 4.

\section{Discussion}

To our knowledge, this is the first study to systematically investigate the relationship between OSA and POAF in patients who underwent septal myectomy. The main findings of the present study are as follows. First, the prevalence of POAF was significantly higher in patients with OSA and increased with worsening severity of OSA. Second, the AHI value was significantly higher in patients with POAF than in those without POAF among the different OSA groups. Third, in the multivariable analysis, 
Table 3 Comparison of clinical variables between patients with and without POAF

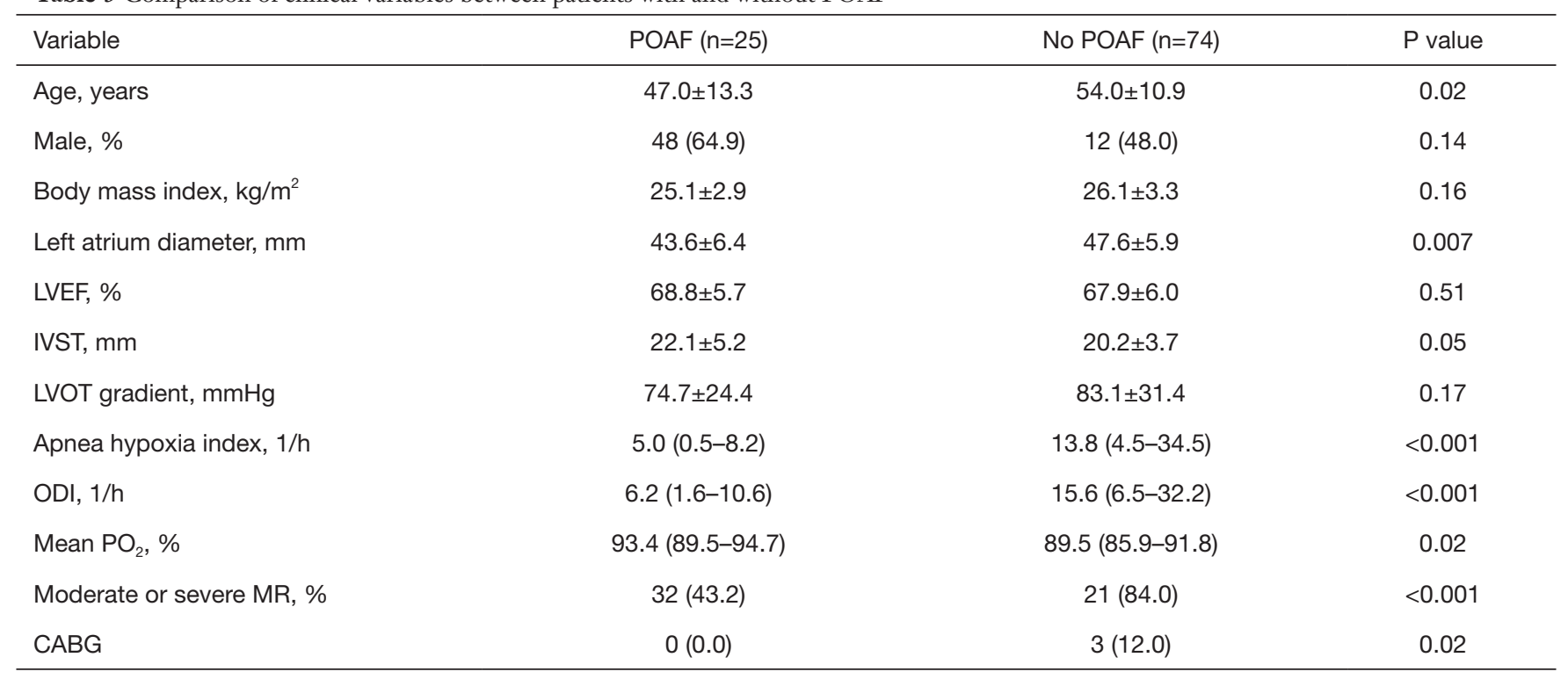

Values are presented as percentage, mean $\pm \mathrm{SD}$, or median (interquartile range) when appropriate. POAF, postoperative atrial fibrillation; LVEF, left ventricular ejection fraction; IVST, interventricular septal thickness; LVOT, left ventricular outflow tract; ODI, oxygen desaturation index; CABG, coronary artery bypass graft.
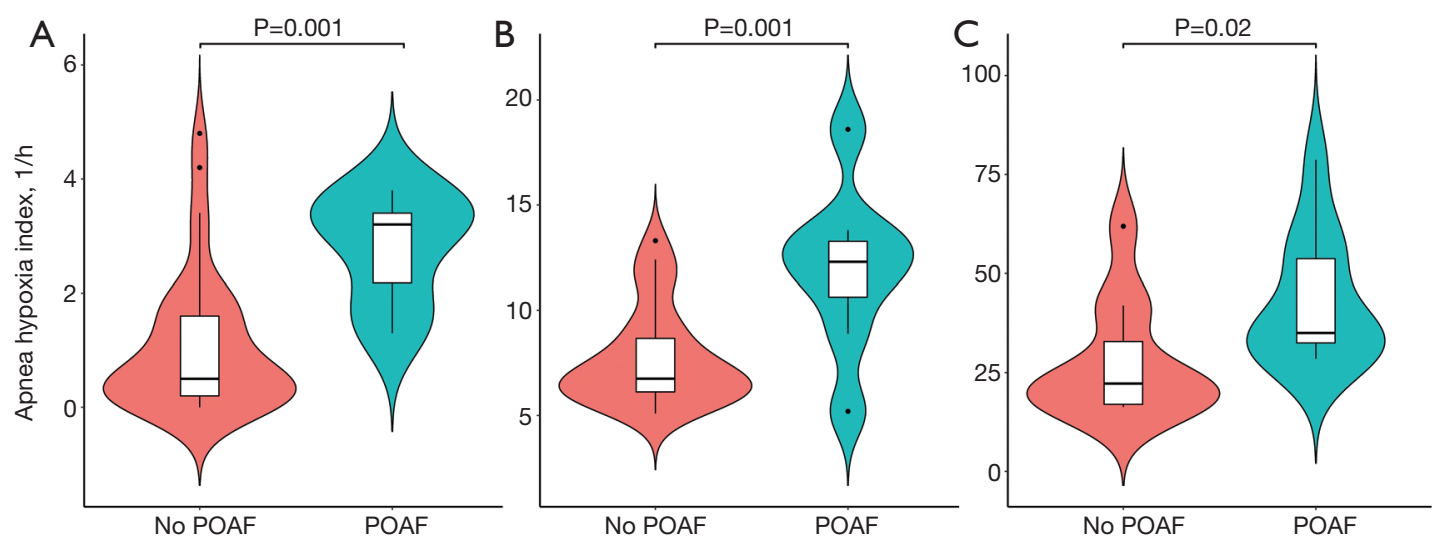

Figure 2 The value of apnea hypopnea index according to the presence of POAF among the different OSA groups. (A) Without OSA. (B) Mild OSA. (C) Moderate-severe OSA. POAF, postoperative atrial fibrillation; OSA, obstructive sleep apnea.

AHI, moderate-to-severe OSA, and left atrium diameter were independent risk factors associated with POAF when adjusted for age, sex, BMI, and other relevant variables from univariable analyses. However, the association between the diagnosis of OSA and POAF was no longer significant.

POAF is a common complication and associated with a longer hospital stay and increased risk of in-hospital stroke after cardiac surgery, including septal myectomy (15). Many factors have been associated with POAF in patients who underwent cardiac surgery, such as age, a history of $\mathrm{AF}$, chronic lung disease, and valve surgery (16). However, few studies have reported factors associated with POAF in patients who underwent septal myectomy. Early and late POAF are independently associated with cardiac mortality, and early POAF occurs in $17-25 \%$ of patients with HOCM who underwent septal myectomy. In the present study, POAF occurred in $25.3 \%$ of all patients during the perioperative period, which is consistent with previous 
Table 4 Logistic analysis for predictors of postoperative atrial fibrillation

\begin{tabular}{|c|c|c|}
\hline \multirow{2}{*}{ Variable } & \multicolumn{2}{|c|}{ Univariable } \\
\hline & OR $(95 \% \mathrm{Cl})$ & $P$ \\
\hline Age, years & $1.05(1.00-1.09)$ & 0.02 \\
\hline Male sex & $2.00(0.80-5.01)$ & 0.14 \\
\hline Body mass index, $\mathrm{kg} / \mathrm{m}^{2}$ & $1.11(0.96-1.29)$ & 0.17 \\
\hline Left atrium diameter, mm & $1.10(1.02-1.19)$ & 0.01 \\
\hline IVST, mm & $0.91(0.82-1.02)$ & 0.09 \\
\hline Moderate or severe MR & $6.89(2.15-22.07)$ & 0.001 \\
\hline ODI, 1/h & $1.05(1.02-1.09)$ & 0.002 \\
\hline Mean $\mathrm{PO}_{2}, \%$ & $0.88(0.79-0.98)$ & 0.02 \\
\hline Apnea hypoxia index $\geq 10.41 / h$ & $8.36(3.03-23.03)$ & $<0.001$ \\
\hline OSA severity & - & 0.01 \\
\hline No OSA & Reference & \\
\hline Mild OSA & $1.89(0.59-6.12)$ & 0.28 \\
\hline Moderate-severe OSA & $6.17(1.86-20.50)$ & 0.003 \\
\hline \multicolumn{3}{|l|}{ Presence of OSA } \\
\hline No OSA & Reference & \\
\hline OSA & $3.17(1.14-8.82)$ & 0.03 \\
\hline \multicolumn{3}{|l|}{ Model 1} \\
\hline Left atrium diameter & $4.84(1.41-16.56)$ & 0.01 \\
\hline Apnea hypoxia index $\geq 10.41 / \mathrm{h}$ & $6.29(2.18-18.14)$ & 0.001 \\
\hline Moderate or severe MR & $1.09(1.00-1.19)$ & 0.05 \\
\hline \multicolumn{3}{|l|}{ Model 2} \\
\hline Left atrium diameter & $1.09(1.00-1.18)$ & 0.04 \\
\hline OSA severity & - & 0.03 \\
\hline No OSA & Reference & \\
\hline Mild OSA & $1.63(0.49-5.42)$ & 0.42 \\
\hline Moderate-severe OSA & $4.88(1.42-16.86)$ & 0.01 \\
\hline \multicolumn{3}{|l|}{ Model 3} \\
\hline Left atrium & $1.12(1.03-1.22)$ & 0.01 \\
\hline
\end{tabular}

Model 1: The value of $\mathrm{AHI}$ was entered into the model, and the model was adjusted for variables from univariate analysis; Model 2: The severity of OSA was entered into the model, and the model was adjusted for variables from univariate analysis; Model 3: The presence of OSA was entered into the model, and the model was adjusted for variables from univariate analysis. IVST, interventricular septal thickness; ODI, oxygen desaturation index; CABG, coronary artery bypass graft; OSA, obstructive sleep apnea. studies $(4,17)$. In addition, studies have shown that OSA is an independent risk factor for POAF in patients undergoing cardiac surgery, including coronary artery bypass grafting, valve replacement/repair, and combined valve/coronary artery bypass grafting $(5,18,19)$.

OSA may affect the heart rhythm through many mechanisms, including activation of the sympathetic nervous system, increased serum inflammatory cytokine levels, cardiac remodeling, insulin resistance, and impairment of vascular endothelial function (20). In our study, we found that the levels of fasting glucose, high-sensitivity C-reactive protein, and big endothelin were significantly higher in patients with OSA, which is consistent with previous studies. These results showed that the levels of inflammation and insulin resistance were higher in patients with OSA. All of these factors may contribute to the development of POAF in these patients. Furthermore, the prevalence of moderate or severe mitral regurgitation and left atrial diameter were significantly higher in patients with HOCM complicated with OSA. The likelihood of mitral valve intervention is relatively high in these patients, which might also be a risk factor for POAF. Futile inspiratory efforts caused by OSA increase left ventricular transmural pressure, and hence the afterload might also increase. All of these factors may result in cardiac remodeling, including atrial and ventricular remodeling (21). In addition, OSA is associated with electrophysiological changes in the atrium, such as a reduction in voltage, conduction slowing, and longer sinus node recovery (22). Importantly, we found that AHI was significantly higher in patients with POAF among the different OSA groups and increased with worsening severity of OSA. These results suggest that nonhypertrophic cardiomyopathy-related comorbidities may play a role in POAF development.

Many factors might be associated with POAF in patients who underwent septal myectomy, including age, sex, and mitral intervention. Therefore, we conducted univariate analysis to confirm the variables associated with POAF in these patients. We found that left atrium diameter, interventricular septal thickness, and moderate or severe mitral regurgitation were risk factors for POAF. Importantly, the AHI, severity of OSA, and presence of OSA were also associated with POAF. However, when adjusted for age, sex, body mass index, and other relevant variables, only left atrium diameter, AHI, and moderateto-severe OSA were independent risk factors associated with POAF instead of OSA. The findings of the present 
study suggest that the severity of OSA might increase the prevalence of POAF and might provide some new information about the relationship between OSA and POAF in patients with HOCM who underwent septal myectomy.

POAF was associated with adverse clinical outcomes after septal myectomy in patients with HOCM, which was similar to that seen in patients with preoperative $\mathrm{AF}$ (4). In addition, patients with severe untreated OSA had a higher incidence of fatal and non-fatal cardiovascular events than those without OSA; however, the exact mechanism is unclear. OSA is a syndrome that can be relieved with continuous positive airway pressure therapy. A previous study reported that preoperative treatment of OSA using continuous positive airway pressure therapy was associated with a decreased rate of POAF after cardiac surgery (18). In patients with HOCM complicated with OSA, application of continuous positive airway pressure is apparently safe and does not lead to hemodynamic compromise (23). Therefore, we think that early diagnosis and timely treatment with continuous positive airway pressure therapy for these patients will reduce the incidence of POAF caused by more severe OSA and might improve the long-term clinical outcomes in patients with HOCM who underwent septal myectomy.

\section{Limitations}

There are some limitations to our study. First, this was a single-center study, and the number of patients was small. A multicenter study with a large population of patients with HOCM who underwent septal myectomy is needed to confirm our results, and a multicenter collaborative clinical trial study on the treatment effects of OSA on POAF is necessary. Second, factors associated with POAF in patients with HOCM who underwent septal myectomy are complex, and the present study provides only some information for a new perspective on this topic. Third, all patients in the present study completed only one night of sleep study. OSA-related variables show considerable night-to-night variability, and single-night diagnostic sleep studies are prone to mis-categorization of the severity of OSA.

\section{Conclusions}

The severity of OSA reflected by the AHI in patients with HOCM who underwent septal myectomy is an independent risk factor for POAF, which is associated with adverse clinical outcomes. The present results suggest that more attention should be paid and treatment decisions should be made in patients with HOCM who underwent septal myectomy complicated with moderate or severe OSA. Further studies are needed to investigate the effect of OSA on future cardiovascular complications, as well as the safety and efficacy of OSA treatment in these patients.

\section{Acknowledgments}

We would like to thank Editage for their help in polishing our paper.

Funding: This work was supported by National Natural Science Foundation of China (Grant No. 81770371).

\section{Footnote}

Reporting Checklist: The authors have completed the STROBE reporting checklist. Available at https://dx.doi. org/10.21037/jtd-21-632

Data Sharing Statement: Available at https://dx.doi. org/10.21037/jtd-21-632

Conflicts of Interest: All authors have completed the ICMJE uniform disclosure form (available at https://dx.doi. org/10.21037/jtd-21-632). The authors have no conflicts of interest to declare.

Ethical Statement: The authors are accountable for all aspects of the work in ensuring that questions related to the accuracy or integrity of any part of the work are appropriately investigated and resolved. The study was conducted in accordance with the Declaration of Helsinki (as revised in 2013). The study was approved by the Institutional Review Board of Anzhen Hospital (Ethic committee study number: 2020034X), which is affiliated with Capital Medical University (Beijing, China), and individual consent for this retrospective analysis was waived.

Open Access Statement: This is an Open Access article distributed in accordance with the Creative Commons Attribution-NonCommercial-NoDerivs 4.0 International License (CC BY-NC-ND 4.0), which permits the noncommercial replication and distribution of the article with the strict proviso that no changes or edits are made and the original work is properly cited (including links to both the formal publication through the relevant DOI and the license). See: https://creativecommons.org/licenses/by-nc-nd/4.0/. 


\section{References}

1. Woldendorp K, Farag J, Khadra S, et al. Postoperative atrial fibrillation after cardiac surgery: a meta-analysis. Ann Thorac Surg 2020. [Epub ahead of print]. doi: 10.1016/ j.athoracsur.2020.10.055.

2. Ommen SR, Maron BJ, Olivotto I, et al. Long-term effects of surgical septal myectomy on survival in patients with obstructive hypertrophic cardiomyopathy. J Am Coll Cardiol 2005;46:470-6.

3. Guo Y, Wu X, Zheng X, et al. Usefulness of Preoperative Transforming Growth Factor-Beta to Predict New Onset Atrial Fibrillation After Surgical Ventricular Septal Myectomy in Patients With Obstructive Hypertrophic Cardiomyopathy. Am J Cardiol 2017;120:118-23.

4. Tang B, Song Y, Cheng S, et al. In-Hospital Postoperative Atrial Fibrillation Indicates a Poorer Clinical Outcome after Myectomy for Obstructive Hypertrophic Cardiomyopathy. Ann Thorac Cardiovasc Surg 2020;26:22-9.

5. Foldvary-Schaefer N, Kaw R, Collop N, et al. Prevalence of Undetected Sleep Apnea in Patients Undergoing Cardiovascular Surgery and Impact on Postoperative Outcomes. J Clin Sleep Med 2015;11:1083-9.

6. Nagappa M, Ho G, Patra J, et al. Postoperative Outcomes in Obstructive Sleep Apnea Patients Undergoing Cardiac Surgery: A Systematic Review and Meta-analysis of Comparative Studies. Anesth Analg 2017;125:2030-7.

7. Wong JK, Maxwell BG, Kushida CA, et al. Obstructive Sleep Apnea Is an Independent Predictor of Postoperative Atrial Fibrillation in Cardiac Surgery. J Cardiothorac Vasc Anesth 2015;29:1140-7.

8. Wang S, Cui H, Meng L, et al. Effect of Septal Myectomy on Obstructive Sleep Apnoea Syndrome in Patients With Hypertrophic Obstructive Cardiomyopathy. Heart Lung Circ 2020;29:1366-74.

9. Konecny T, Brady PA, Orban M, et al. Interactions between sleep disordered breathing and atrial fibrillation in patients with hypertrophic cardiomyopathy. Am J Cardiol 2010;105:1597-602.

10. American College of Cardiology Foundation/American Heart Association Task Force on Practice; American Association for Thoracic Surgery; American Society of Echocardiography, et al. 2011 ACCF/AHA guideline for the diagnosis and treatment of hypertrophic cardiomyopathy: a report of the American College of Cardiology Foundation/American Heart Association Task
Force on Practice Guidelines. J Thorac Cardiovasc Surg 2011;142:e153-203.

11. Elliott PM, Anastasakis A, Borger MA, et al. 2014 ESC Guidelines on diagnosis and management of hypertrophic cardiomyopathy: the Task Force for the Diagnosis and Management of Hypertrophic Cardiomyopathy of the European Society of Cardiology (ESC). Eur Heart J 2014;35:2733-79.

12. Lang RM, Bierig M, Devereux RB, et al. Recommendations for chamber quantification: a report from the American Society of Echocardiography's Guidelines and Standards Committee and the Chamber Quantification Writing Group, developed in conjunction with the European Association of Echocardiography, a branch of the European Society of Cardiology. J Am Soc Echocardiogr 2005;18:1440-63.

13. Morgenthaler TI, Aurora RN, Brown T, et al. Practice parameters for the use of autotitrating continuous positive airway pressure devices for titrating pressures and treating adult patients with obstructive sleep apnea syndrome: an update for 2007. An American Academy of Sleep Medicine report. Sleep 2008;31:141-7.

14. Guo HC, Wang Y, Dai J, et al. Application of 3D printing in the surgical planning of hypertrophic obstructive cardiomyopathy and physician-patient communication: a preliminary study. J Thorac Dis 2018;10:867-73.

15. Eikelboom R, Sanjanwala R, Le ML, et al. Postoperative Atrial Fibrillation After Cardiac Surgery: A Systematic Review and Meta-Analysis. Ann Thorac Surg 2021;111:544-54.

16. Mathew JP, Fontes ML, Tudor IC, et al. A multicenter risk index for atrial fibrillation after cardiac surgery. JAMA 2004;291:1720-9.

17. Desai MY, Bhonsale A, Smedira NG, et al. Predictors of long-term outcomes in symptomatic hypertrophic obstructive cardiomyopathy patients undergoing surgical relief of left ventricular outflow tract obstruction. Circulation 2013;128:209-16.

18. Wong JK, Mariano ER, Doufas AG, et al. Preoperative Treatment of Obstructive Sleep Apnea With Positive Airway Pressure is Associated With Decreased Incidence of Atrial Fibrillation After Cardiac Surgery. J Cardiothorac Vasc Anesth 2017;31:1250-6.

19. Mungan U, Ozeke O, Mavioglu L, et al. The role of the preoperative screening of sleep apnoea by Berlin Questionnaire and Epworth Sleepiness Scale for postoperative atrial fibrillation. Heart Lung Circ 
2013;22:38-42.

20. Lavergne F, Morin L, Armitstead J, et al. Atrial fibrillation and sleep-disordered breathing. J Thorac Dis 2015;7:E575-84.

21. Bradley TD, Floras JS. Sleep apnea and heart failure: Part I: obstructive sleep apnea. Circulation 2003;107:1671-8.

22. Dimitri H, Ng M, Brooks AG, et al. Atrial remodeling in

Cite this article as: Yu J, Wang S, Ren C, Guo H, Ma H, Wei Z, Lai Y. Impact of obstructive sleep apnea on new-set atrial fibrillation after septal myectomy in patients with hypertrophic obstructive cardiomyopathy. J Thorac Dis 2021;13(8):4915-4924. doi: $10.21037 /$ jtd-21-632 obstructive sleep apnea: implications for atrial fibrillation. Heart Rhythm 2012;9:321-7.

23. Nerbass FB, Salemi VMC, Pedrosa RP, et al. Acute Effects of Nasal CPAP in Patients With Hypertrophic Cardiomyopathy. Chest 2016;150:1050-8. 